\title{
Tortuosidad y permeabilidad de materiales cerámicos mesoporosos de caolín y diatomita
}

\section{Tortuosity and permeability of mesoporous ceramics of kaolin and diatomite}

\author{
Luis A. Fonseca ${ }^{1}$, Emanuel Trujillo-Castro ${ }^{2 a}$, Gabriel Peña-Rodríguez $^{2 b}$ \\ ${ }^{1}$ Doctorado en Ingeniería y Ciencia de los Materiales, Universidad Pedagógica y Tecnológica de Colombia, Colombia. \\ Correo electrónico: alejandro.fonseca@uptc.edu.co \\ ${ }^{2}$ Grupo de Investigación en Instrumentación y Física de la Materia Condensada (Gifimac), Universidad Francisco de Paula \\ Santander, Colombia. Correo electrónico: aemanueltca@ufps.edu.co, bgabrielpr@ufps.edu.co
}

Recibido: 8 noviembre, 2017. Aceptado: 25 abril, 2018. Versión final: 22 junio, 2018.

\begin{abstract}
Resumen
Se reporta la permeabilidad y tortuosidad de cerámicas mesoporosas de caolines y diatomitas. Las muestras cilíndricas con porcentaje en peso de $60 \%$ diatomita, $30 \%$ caolín y $10 \% \mathrm{CaCO}_{3}$, fueron elaboradas por colado, con diámetro promedio de $5 \mathrm{~cm}$ y espesor de $0,6 \mathrm{~cm}$. El proceso de secado se realizó mediante estufa de circulación forzada a $100{ }^{\circ} \mathrm{C}$ por 24 horas, la sinterización a temperaturas de $800{ }^{\circ} \mathrm{C}, 850{ }^{\circ} \mathrm{C}, 900{ }^{\circ} \mathrm{C}, 950{ }^{\circ} \mathrm{C}, 1.000{ }^{\circ} \mathrm{C}$ y $1.100{ }^{\circ} \mathrm{C}$, usando una mufla eléctrica con una rampa de $5{ }^{\circ} \mathrm{C} / \mathrm{min}$. Para la determinación de permeabilidad por el agua, se utilizó un permeámetro de carga variable, mientras que la tortuosidad se halló del producto del factor de forma y su porosidad. Se encontraron resultados de tortuosidad en el rango de 3,32 a 7,16, mientras que la permeabilidad varió entre 3,09x $10^{-}$ ${ }^{6}$ y $22,2 \times 10^{-6} \mathrm{~cm} / \mathrm{s}$, evidenciándose un comportamiento inverso entre estos parámetros al incrementarse la temperatura.
\end{abstract}

Palabras clave: cerámica mesoporosa; factor de forma; permeabilidad; resistividad eléctrica; tortuosidad.

\begin{abstract}
In this paper, the permeability and tortuosity of mesoporous ceramics of kaolin and diatomite are reported. The cylindrical samples with weight percentages of $60 \%$ diatomite, $30 \%$ kaolin and $10 \% \mathrm{CaCO} 3$ were made by casting, with an average diameter of $5 \mathrm{~cm}$ and a thickness of $0.6 \mathrm{~cm}$. The drying process was carried out through the forced circulation stove at $100{ }^{\circ} \mathrm{C}$ for 24 hours and the sintering at temperatures of $800{ }^{\circ} \mathrm{C}, 850{ }^{\circ} \mathrm{C}, 900{ }^{\circ} \mathrm{C}, 950{ }^{\circ} \mathrm{C}, 1000{ }^{\circ} \mathrm{C}$ and $1100^{\circ} \mathrm{C}$, using an electric muffle with a ramp of $5^{\circ} \mathrm{C} / \mathrm{min}$. For the determination of water permeability, a variable load permeameter was used, while the tortuosity was found from the product of the form factor and its porosity. The results of tortuosity were found in the range of 3.32 to 7.16 , whereas permeability varied between $3.09 \times 10-6$ and $22.2 \times 10-6 \mathrm{~cm} / \mathrm{s}$, evidencing an inverse behavior between these parameters when increasing to temperature.
\end{abstract}

Keywords: form factor; tortuosity; permeability; electrical resistivity; mesoporous ceramics.

\section{Introducción}

La tortuosidad y permeabilidad son parámetros importantes para representar la complejidad estructural de un medio poroso, cuando se quiere estudiar los fenómenos de transporte a través de estos $[1,2,3]$.

Desde el punto de vista teórico (ecuación de Kozeny

ISSN impreso: 1657 - 4583, ISSN en línea: 2145 - 8456, CC BY-ND 4.0 (c) (i)

Como citar: L. A. Fonseca, E. Trujillo-Castro, G. Peña-Rodríguez, "Tortuosidad y permeabilidad de materiales cerámicos mesoporosos de caolín y diatomita," Rev. UIS Ing., vol. 18, no. 1, pp. 111-118, 2019. doi: 10.18273/revuin.v18n1-2019009 
112 ㅈUㄴ REVISTA UIS

Carman, ley de Archie), la permeabilidad depende del factor de porosidad, de forma y de tortuosidad $[4,5]$. La tortuosidad es un concepto físico que puede interpretarse como la razón entre la trayectoria seguida por un flujo al atravesar un medio poroso y el espesor de ese material [6, 7].

Desde el punto de vista experimental, se han desarrollado varios métodos para determinar la tortuosidad, donde se han aplicado técnicas electroquímicas, de ultrasonido, hidráulicas, eléctricas y de simulación, entre otras $[8,9$, $10]$.

En los fenómenos de transporte a través de medios porosos, debido a la existencia de un gradiente, térmico, de concentración, de masa o de presión, es importante la predicción en tiempo real de las propiedades térmicas, acústicas y eléctricas. Para lo cual se hace uso de algoritmos y modelado de los flujos que atraviesan ese medio, donde se emplean métodos de espiral, vórtices, pesos ponderados y electromagnéticos, entre otros [11].

En las cerámicas, durante el proceso de sinterización, la porosidad intergranular es eliminada vía difusión atómica, al incrementarse la temperatura, de modo que el tamaño del poro crece al aumentar la sinterización, aunque se produce una reducción de la porosidad total en el material [12]. De lo anterior se desprende que las cerámicas, aunque sean frágiles, poseen buena resistencia mecánica y térmica, pero, debido a su porosidad, son ampliamente usadas en procesos de filtración, separación de fluidos, catálisis, intercambiadores de calor, remediación de suelos y muchas aplicaciones tecnológicas propias de los fenómenos de transporte [7, $13,14]$.

Por lo anterior se reporta la permeabilidad al agua y la tortuosidad de cerámicas mesoporosas, elaboradas a base de arcillas caolinitas y tierras diatomáceas, sinterizadas a temperaturas en un rango de $800{ }^{\circ} \mathrm{C}$ y $1.100{ }^{\circ} \mathrm{C}$, con el objeto de estudiar el efecto de la temperatura de cocción en estos parámetros. Para hallar la tortuosidad, se implementó un sistema automatizado, que permite tener la medida del factor de forma $(\mathrm{F})$, mientras que, para determinar la permeabilidad por el agua, se construyó un permeámetro de carga variable.

\section{Materiales y métodos}

\subsection{Los cerámicos porosos}

Las cerámicas porosas utilizadas en este trabajo fueron fabricadas utilizando el proceso de colado en moldes de yeso escayola. La mezcla utilizada para la fabricación de las muestras en cuanto a su peso fueron $60 \%$ de caolín,
$30 \%$ de diatomita y $10 \%$ de carbonato de calcio. El proceso de secado se realizó a temperatura ambiente durante un tiempo de 24 horas, posteriormente se utilizó una estufa de circulación forzada a una temperatura de $100{ }^{\circ} \mathrm{C}$ por un lapso igual. El proceso de cocción se realizó mediante una mufla eléctrica en un rango de temperaturas de $800{ }^{\circ} \mathrm{C}$ y $1.100{ }^{\circ} \mathrm{C}$, con el objeto de generar diferentes porosidades en la muestra.

\subsection{Porosidad total}

La porosidad total de un medio poroso está definida de la siguiente manera $[15,16]$ :

$\varepsilon=\frac{\text { Volumen del vacío }}{\text { Volumento total de la muestra }}=\frac{v_{F}}{v_{F}}$

El volumen del vacío $\left(V_{F}\right)$ es llenado por medio del fluido que se aloja en su interior, pero no todo este líquido fluye cuando se le aplica una diferencia de presión. Una parte de este líquido se mantiene alojado y no aporta a la velocidad del flujo del fluido. La porosidad efectiva, es decir, la que contribuye al flujo del líquido, se define así $[15,16]$ :

$\varepsilon^{\prime}=\frac{\text { Volumen total de poros abiertos }}{\text { Volumen aparente de la muestra }}=\frac{\mathrm{V}_{\mathrm{Ta}}}{\mathrm{V}_{\mathrm{ap}}}$

La porosidad total de un medio poroso puede ser medida mediante la ecuación (3) [16].

$\varepsilon=1-\frac{\rho_{\mathrm{a}}}{\rho_{\mathrm{r}}}$

Dónde $\rho_{\mathrm{a}}$ es la densidad aparente de la muestra (masa en seco de la muestra/volumen total de la muestra) y $\rho_{\mathrm{r}}$ es la densidad absoluta del medio, medida aquí mediante la técnica del picnómetro.

\subsection{Tortuosidad del medio poroso}

Sheidegger [17] definió la tortuosidad como la relación entre la longitud real que recorre el fluido en un medio poroso $\left(L_{h}\right)$ y el espesor del medio $\left(L_{s}\right)$. Pero esta misma se puede obtener mediante el producto del factor de forma $(F)$ y la porosidad total $(\varepsilon)$, donde $F$ está dada como el cociente de la resistividad eléctrica de la muestra completamente saturada con una solución conductora $\left(\delta_{\mathrm{m}}\right)$ y la resistividad del líquido de saturación $\left(\delta_{\mathrm{s}}\right)$, como lo muestra la ecuación (4) $[18,19,20]$.

$F=\frac{\delta_{\mathrm{m}}}{\delta_{\mathrm{s}}}$ 
Por lo tanto, la tortuosidad se define de la siguiente manera $[19,20,21]$ :

$\tau=\varepsilon F$

En la figura 1, se presenta el diagrama experimental usado para determinar $\mathrm{F}$, el cual consta de una cámara portamuestra elaborada de polietileno (HDPE), que aloja los dos electrodos de $\mathrm{Ag} / \mathrm{AgCl}$, conectados al sistema de acondicionamiento de señal, que consta de una tarjeta de adquisición de datos y una fuente de corriente, que envían la información a la interfaz del usuario. La muestra porosa se deposita en la cámara, seguidamente se inyecta un electrolito $\left(0,1 \mathrm{~mol} \mathrm{H}_{2} \mathrm{SO}_{4}\right)$ de resistividad eléctrica conocida $\delta_{s}=13.24 \Omega \cdot \mathrm{m}$, a través de los orificios donde se sitúan los electrodos, y se deja saturar la muestra por un lapso de 24 horas, tiempo seguido se ponen los electrodos. Posteriormente se aplica una corriente de 2 $\mathrm{mA}$ en los extremos laterales de la portamuestras, y se inicia la lectura de la diferencia de potencial a través de los electrodos, señal que es leída por el microcontrolador y es enviada vía comunicación bluetooth a la interfaz del usuario.

En la figura 2, se observa el diagrama general de la tarjeta de adquisición de datos, que tiene como etapa inicial un oscilador de cambio de fase. En seguida, se encuentra un amplificador de instrumentación, que se encarga de recibir la diferencia de potencial proveniente de los electrodos de $\mathrm{Ag} / \mathrm{AgCl}$ y de amplificar este voltaje que se encuentra en el rango de los milivoltios. Como etapa de acondicionamiento de señal se encuentran un filtro de paso bajo y uno de paso alto, que son los encargados de evitar señales de ruido presentes en el ambiente. Después, la señal pasa a través de un amplificador sumador que se encarga de elevar la señal. Por último, la señal es recibida por el microcontrolador, que se encarga de tratar los datos y enviarlos vía bluetooth, cada vez que el usuario lo requiera, por medio de la interfaz de usuario desde el ordenador. Con los datos anteriores se determina la resistividad de la muestra saturada $\boldsymbol{\delta}_{\boldsymbol{m}}$, y, usando las ecuaciones (4) y (5), se halla el factor F y la tortuosidad $(\tau)$ de cada muestra.

\subsection{Permeabilidad}

Hace referencia a la capacidad que posee una estructura o material a ser atravesada por un fluido, sin que este modifique su aspecto. Se puede clasificar como permeable cuando un fluido logra atravesar esa composición, y, por el contrario, se le denomina impermeable a toda estructura que impide el paso de un fluido.

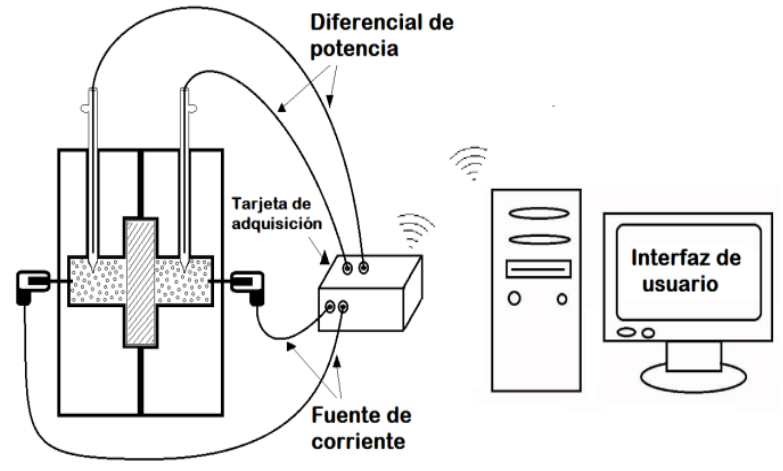

Figura 1. Diagrama esquemático del equipo para la medicion factor de forma $(F)$. Fuente: elaboración propia.

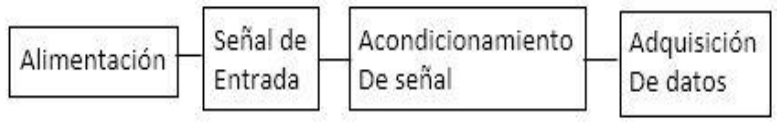

Figura 2. Diagrama de bloques de tarjeta de adquisición de datos. Fuente: elaboración propia.

La permeabilidad de un material depende de factores como la porosidad, la densidad, la presión y la temperatura del fluido [22]

En la figura 3, se presenta el esquema experimental del método de carga variable, el cual es ampliamente usado para hallar la permeabilidad en medios porosos [23]. El modelo consiste en medir la cantidad de agua filtrada durante un tiempo específico, sin adicionar fluido.

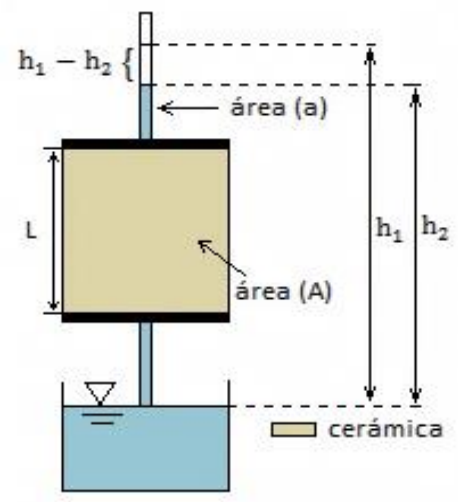

Figura 3. Permeámetro de carga variable. Fuente: elaboración propia.

El coeficiente de permeabilidad $(k)$ es expresado mediante la ecuación (6) [24].

$k=2,3 \frac{a \times L}{A \times \Delta t} \times \log _{10}\left(\frac{h_{1}}{h_{2}}\right)$ 
Donde,

$a=$ área del tanque

$L=$ espesor de la muestra

$\Delta t=$ tiempo de muestreo

$A=$ área de la muestra

$h_{1}=$ nivel inicial de agua

$h_{2}=$ nivel final de agua

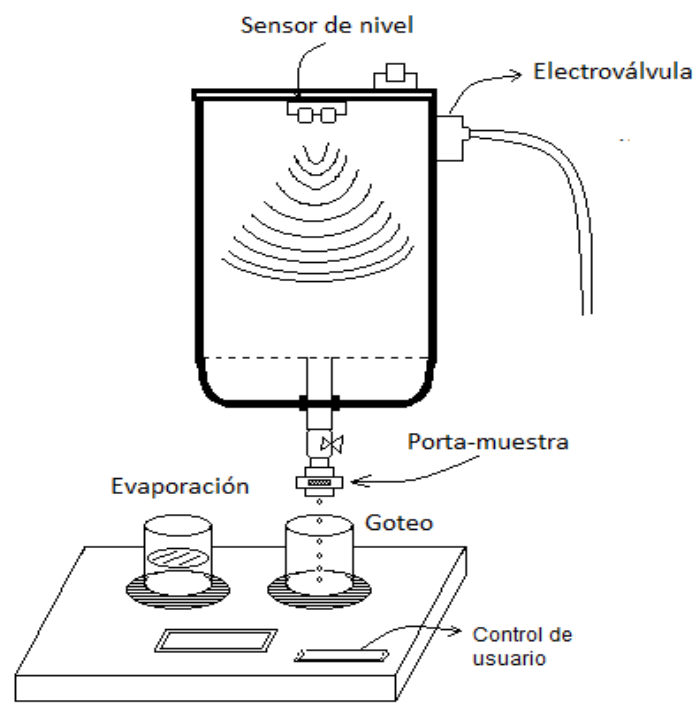

Figura 4. Esquemático de equipo para la medición de permeabilidad. Fuente: elaboración propia.

Las mediciones de permeabilidad fueron realizadas empleando el equipo observado en la figura 4. Para las pruebas, el tiempo de muestreo fue de 24 horas. Las dimensiones de las muestras utilizadas fueron $3,6 \mathrm{~cm}$ de diámetro y un máximo de espesor de $0,6 \mathrm{~cm}$.

El sistema está conformado por un par de celdas de carga, las cuales se encargan de medir la masa de agua que es filtrada a través del medio poroso y la que es evaporada durante el tiempo de muestreo por causa de la temperatura ambiente. Como etapa de adquisición de datos cuenta con una tarjeta Arduino, que almacena los datos en función del tiempo en una memoria SD. Los resultados son analizados mediante la interfaz de usuario diseñada para el sistema.

El procedimiento experimental consiste en el llenado del tanque hasta su nivel establecido, seguido de ello se ingresa la muestra en el portamuestra y se da inicio a la medición. Una vez transcurrido el tiempo de muestreo, se extraen los datos y se presenta el coeficiente de permeabilidad por medio de la interfaz de usuario del sistema.

\section{Resultados y discusión}

\subsection{Medición experimental de tortuosidad}

En la tabla 1, se reportan los datos de $\boldsymbol{\delta}_{\boldsymbol{m}}, \boldsymbol{\varepsilon}, \mathrm{F}$ y de la tortuosidad $(\tau)$ de las muestras para cada temperatura de sinterización. Se observa que, al aumentar la temperatura de cocción en el rango entre 800 y $1.100{ }^{\circ} \mathrm{C}$, la porosidad total no presentó variaciones significativas, y reportó valores entre 0,54 y 0,60 . Sin embargo, el factor de forma decrece, lo cual implica una reducción de la resistividad eléctrica en las muestras al aumentar la temperatura de cocción. Por último, sobre la tortuosidad, cuyos valores se encuentran en un rango de 3,32 y 7,16, se observa que, a mayor temperatura, menor es su tortuosidad, lo cual permite un mayor paso del fluido en el medio poroso.

Tabla 1. Resultados de la resistividad, porosidad, factor de forma y tortuosidad de las muestras.

\begin{tabular}{c|cccc}
\hline $\begin{array}{c}\text { Temp } \\
\left({ }^{\circ} \mathbf{C}\right)\end{array}$ & $\boldsymbol{\delta}_{\boldsymbol{m}}$ & $\boldsymbol{\varepsilon}$ & $\boldsymbol{F}$ & $\boldsymbol{\tau}$ \\
\hline $\mathbf{8 0 0} \pm \mathbf{8}$ & $167.70 \pm 8$ & $0.56 \pm 0.02$ & $12.67 \pm 0.4$ & $7.16 \pm 0.2$ \\
$\mathbf{8 5 0} \pm \mathbf{8}$ & $127.75 \pm 6$ & $0.6 \pm 0.02$ & $9.65 \pm 0.5$ & $5.78 \pm 0.3$ \\
$\mathbf{9 0 0} \pm \mathbf{9}$ & $117 \pm 6$ & $0.57 \pm 0.02$ & $8.84 \pm 0.4$ & $5.1 \pm 0.3$ \\
$\mathbf{9 5 0} \pm \mathbf{1 0}$ & $98.56 \pm 5$ & $0.58 \pm 0.02$ & $7.44 \pm 0.4$ & $4.33 \pm 0.2$ \\
$\mathbf{1 0 0 0} \pm \mathbf{1 0}$ & $81.77 \pm 4$ & $0.54 \pm 0.02$ & $6.18 \pm 0.3$ & $3.32 \pm 0.1$ \\
\hline
\end{tabular}

Fuente: elaboración propia.

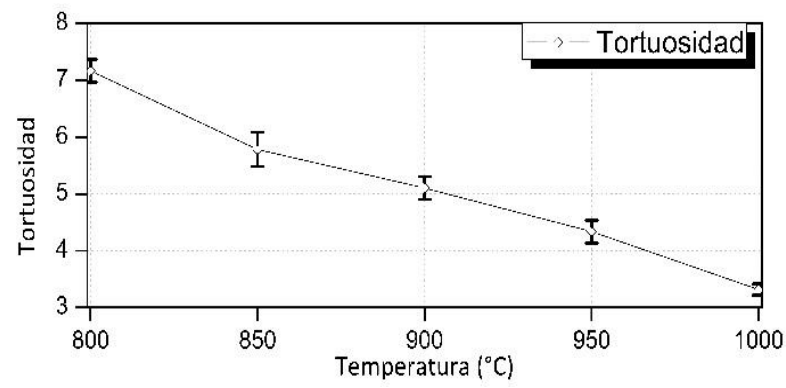

Figura 5. Resultado de tortuosidad en función de temperatura de sinterización. Fuente: elaboración propia.

En la figura 5, se aprecia una disminución de la tortuosidad, al aumentar la temperatura de sinterización, debido a una mayor conectividad de los poros en su estructura interna, lo cual permite una mayor fluidez del líquido, y da como resultado un material menos tortuoso. Este resultado está acorde con lo presentado en la figura 6 , donde se obervan las imágenes de la morfología de las muestras usando microscopía electrónica de barrido (MEB). 


\subsection{Medición experimental de la permeabilidad}

En la figura 6, se presentan microfotografías a $3000 \mathrm{X}$ de la morfología de las muestras usandoMEB. En esta se observa la presencia de las frústulas de las diatomitas, las cuales son altamente porosas. Asimismo, se aprecia que a mayor temperatura de cocción en la muestra, el proceso de sinterización permite definir la porosidad y las paredes de la cerámica porosa, y esto trae como consecuencia un incremento en la densidad de la muestra como consecuencia del proceso de sinterizado.
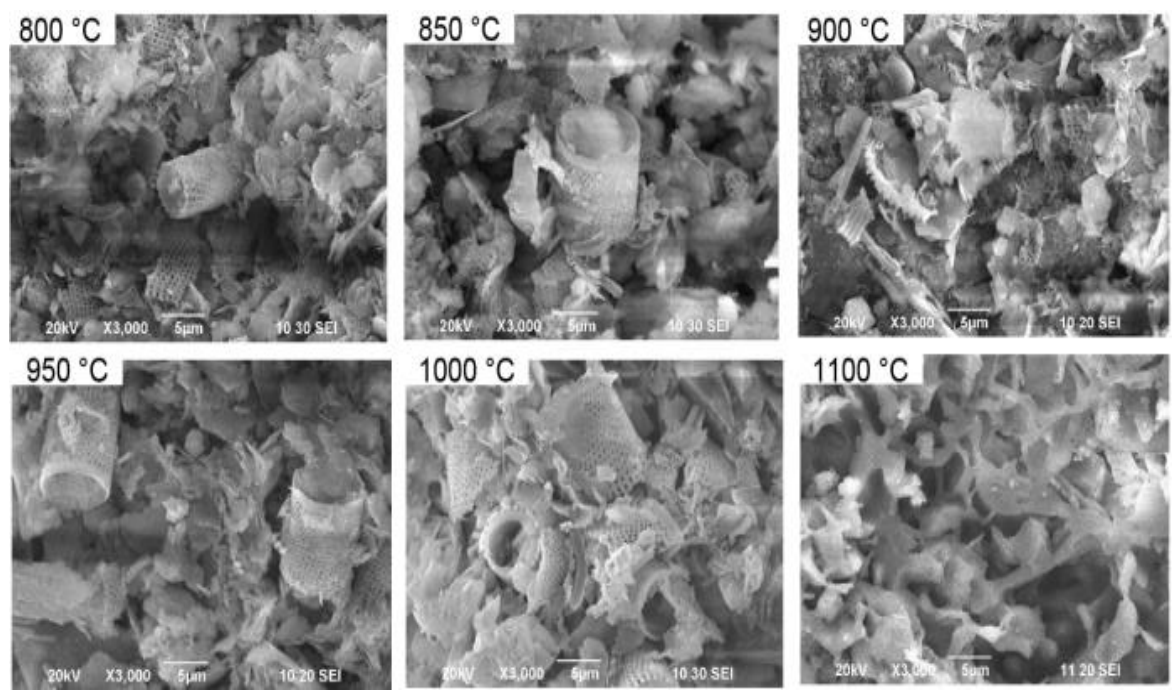

Figura 6. Microfotografías de las cerámicas porosas, usando MEB a 3000X. Fuente: Centro de Investigación en Ciencia Aplicada y Tecnología Avanzada del IPN-México.

El comportamiento de la permeabilidad por el agua en función de la temperatura de cocción de cada una de las muestras se presenta en la figura 7 , donde se grafica la masa de agua que atraviesa la muestra en función del tiempo. Se observa que las muestras con tratamiento térmico de $900{ }^{\circ} \mathrm{C}, 950{ }^{\circ} \mathrm{C}$ y $1.000{ }^{\circ} \mathrm{C}$ tienen un comportamiento similar, a diferencia de la muestra sometida a $1.100{ }^{\circ} \mathrm{C}$, la cual presenta un incremento drástico en su pendiente. Lo anterior se justifica en los resultados encontrados para la permeabilidad de las muestras, los cuales se presentan en la tabla 2. En estos resultados se observa que la muestra sometida a $850{ }^{\circ} \mathrm{C}$ es la que presenta el menor valor, mientras que la tratada a $1.100{ }^{\circ} \mathrm{C}$ reporta el mayor valor. Lo anterior es consistente con lo observado en la morfología usando MEB (ver Figura 6), donde se corrobora que la muestra sometida a $1.100^{\circ} \mathrm{C}$ es la que presenta una porosidad más definida e interconectada, lo que contribuye al incremento de la permeabilidad, debido a la formación de trayectorias menos tortuosas y más permeables al flujo de agua.

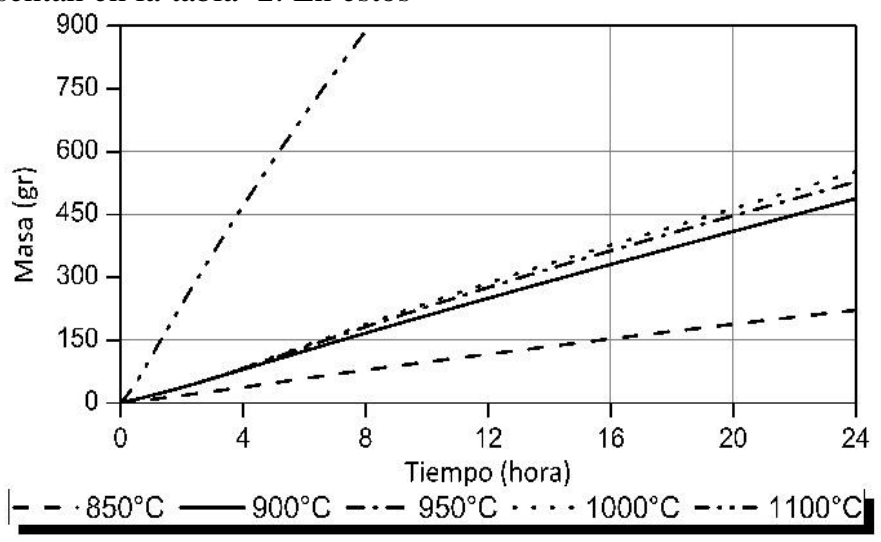

Figura 7. Medida de masa en función del tiempo de fluido filtrado mediante muestra cerámica porosa.

Fuente: elaboración propia. 
Tabla 2. Datos de medida de permeabilidad por el agua en cerámicas de sílice.

\begin{tabular}{c|c}
\hline $\begin{array}{c}\text { Temperatura } \\
\left({ }^{\circ} \mathbf{C}\right)\end{array}$ & $\begin{array}{c}\boldsymbol{K} \\
(\mathbf{c m} / \mathbf{s})\end{array}$ \\
\hline $\mathbf{8 0 0}$ & - \\
$\mathbf{8 5 0}$ & $(3.09 \pm 0.05) \times 10^{-06}$ \\
$\mathbf{9 0 0}$ & $(5.97 \pm 0.09) \times 10^{-06}$ \\
$\mathbf{9 5 0}$ & $(6.92 \pm 0.10) \times 10^{-06}$ \\
$\mathbf{1 0 0 0}$ & $(7.27 \pm 0.11) \times 10^{-06}$ \\
$\mathbf{1 1 0 0}$ & $(22.2 \pm 0.03) \times 10^{-06}$ \\
\hline
\end{tabular}

Fuente: elaboración propia.

Tabla 3. Rangos de permeabilidad [25].

\begin{tabular}{c|c}
\hline $\begin{array}{c}\text { Grado de } \\
\text { Permeabilidad }\end{array}$ & $\begin{array}{c}\text { Valores de } \boldsymbol{K} \\
(\mathbf{c m} / \mathbf{s})\end{array}$ \\
\hline Elevada & $>1 \mathrm{x}^{-01}$ \\
Media & $1 \mathrm{x}^{-01} \mathrm{a} 1 \mathrm{x}^{-03}$ \\
Baja & $1 \mathrm{x}^{-03} \mathrm{a} 1 \mathrm{x}^{-05}$ \\
Muy baja & $1 \mathrm{x}^{-05} \mathrm{a} 1 \mathrm{x}^{-07}$ \\
Practicamente impermeable & $<1 \mathrm{x}^{-07}$ \\
\hline
\end{tabular}

\subsection{Comparación con la literatura}

De los resultados de la tabla 1, se aprecia que, a medida que se incrementa la temperatura de sinterización de la muestra, $\tau$ disminuye. Lo anterior es consecuente con la morfología de las muestras (ver figura 6), donde se observa que, al aumentar la temperatura de sinterización en las muestras, se definen trayectorias menos tortuosas. Por otra parte, al comparar los resultados obtenidos de $\tau$ con los hallados en la literatura [26], se observa que son del mismo orden de magnitud, que reportan valores de $\tau$ entre 3,17 y 4,22, en cerámicas mesoporosas de sílice.

Ademas, al comparar los resultados de permeabilidad, obtenidos a partir del estudio de las muestras (ver tabla 2), con lo informado en la literatura para muestras cerámicas a base de arcillas caoliníticas sinterizadas entre 1.000 y $1.100{ }^{\circ} \mathrm{C}$, que encontraron un valor de $6,33 \times 10^{-5} \mathrm{~cm} / \mathrm{s}$ [27], se afirma que están en el mismo orden de medida, lo cual brinda confiabilidad a los resultados medidos por el sistema diseñado e implementado. Asimismo, al comparar los valores hallados para este parámetro, con el rango de valores reportados para diferentes tipos de medios porosos (ver tabla 3), se observa que las cerámicas de caolín-diatomita se pueden clasificar en el rango de muy baja permeabilidad [25].

\section{Conclusiones}

Se diseñó e implementó un sistema que permite determinar la permeabilidad del material cerámico poroso, mediante el método de carga variable. Para la tortuosidad, se creó un sistema que permite hallar el factor de forma partiendo de principios básicos de la electroquímica, el cual, con la ayuda de la porosidad del material, se obtiene su magnitud.

El análisis de los resultados reporta que, al existir un aumento de temperatura en el proceso de cocción, la permeabilidad del material aumenta y su tortuosidad disminuye. Esto concluye que, a mayor temperatura durante el proceso de sinterización, mayor es el paso del fluido a través del medio poroso.

\section{Referencias}

[1] G. Hai-yan, H. Yue-hui, Z. Jin, X. Nan-ping y C. LIU, "Tortuosity factor for porous $\mathrm{FeAl}$ intermetallics fabricated by reactive synthesis," Transactions of Nonferrous Metals Society of China, vol. 22, pp. 21792183, 2012. doi: 10.1016/S1003-6326(11)61446-5

[2] R. Roque Malherbe, W. del Valle, F. Marquez, J. Duconge y M. Goosen, "Synthesis and Characterization of Zeolite Based Porous Ceramic Membranes," Sep. Sci. Technol., vol. 41, núm. 1, pp. 73-96, ene. 2006. doi: 10.1080/01496390500446277

[3] A. Merioua, A. Bezzar y F. Ghomari, "Electrical prediction of tortuosity in porous media," Energy Procedia, vol. 139, pp. 718-724, 2017. doi: 10.1016/j.egypro.2017.11.277

[4] H. Saomoto y J. Katagiri, "Direct comparison of hydraulic tortuosity and electric tortuosity based on finite element analysis," Theoretical and Applied Mechanics Letters, vol. 5, no. 5pp. 177-180, 2015. doi: 10.1016/j.taml.2015.07.001

[5] K. K. Imomnazarov, “Archie's Law for a Mathematical Model of Movement of a Conducting Liquid through a Conducting Porous Medium," Applied Mathematics Letters, vol. 11, no. 6, pp. 135-138, 1998. doi: 10.1016/S0893-9659(98)00115-3

[6] L. Simao, O. R. Klegues Montedo, M. M. da Silva Paula, L. da Silva, R. Caldato y M. D. de Mello Innocentini, "Structural and Fluid Dynamic Characterization of Calcium Carbonate-based Porous 
Ceramics," Materials Research, vol. 16, no. 4, pp. 14391448, 2013. doi: 10.1590/S1516-14392013005000147

[7] E. A. Moreira y J. R. Coury, "The influence of structural parameters on the permeability of ceramic foams," Brazilian Journal of Chemical Engineering, vol. 21, no. 01, pp. 23-33, 2004. doi: 10.1590/S010466322004000100004

[8] M. Barrande, R. Bouchet y R. Denoyel, "Tortuosity of Porous Particles," Analytical chemistry, vol. 79, no. 23, pp. 9115-9121, 2007. doi: 10.1021/ac071377r

[9] A. Eshghinejadfard, L. Daroczy, G. Janiga y D. Thevenin, "Calculation of the permeability in porous media using the lattice Boltzmann method," International Journal of Heat and Fluid Flow, pp. 1-11, 2016. doi: 10.1016/j.ijheatfluidflow.2016.05.010

[10] Z. Sun, X. Tang y G. Cheng, "Numerical simulation for tortuosity of porous media,"Microporous and Mesoporous Materials, vol. 173, pp. 37-42, 2013. doi: 10.1016/j.micromeso.2013.01.035

[11] E. Garcia, I. Amaya y R. Correa, "Algoritmos de optimización en la estimación de propiedades termodinámicas en tiempo real durante el tratamiento térmico de materiales con microondas," Rev. Uis Ing., vol. 16, no. 2, pp. 129-140, 2017. doi: 10.18273/revuin.v16n2-2017012

[12] Z. Hu y K. Lu, "Evolution of Pores and Tortuosity During Sintering," J. Am. Ceram. Soc., vol. 97, no. 8, pp. 2383-2386, 2014. doi: 10.1111/jace.13080

[13] P. M. Biesheuvel y H. Verweij, "Design of ceramic membrane supports: permeability, tensile strength and stress," Journal of Membrane Science, vol. 156, no. 1, pp. 141-152, 1999. doi: 10.1016/S0376-7388(98)003354

[14] A. Dey, N. Kayal, O. Chakrabarti, R. Caldato, C. André y M. Innocentini, "Permeability and Nanoparticle Filtration Assessment of Cordierite-Bonded Porous SiC Ceramics," Ind. Eng. Chem. Res., vol. 52, no. 51, pp. 18362-18372, 2013. Doi: 10.1021/ie402876v

[15] M. París de Ferrer, Fundamentos de Ingeniería de Yacimientos. Maracaibo, Venezuela: Astro Data, 2009.

[16] Métodos de ensayo para productos refractarios conformados densos. Parte 1: Determinación de la densidad aparente, de la porosidad abierta y de la porosidad total, UNE-EN 993-1, 1996.
[17] A. E. Sheidegger, "Immiscible Multiple Phase Flow", en The Physics of Flow Through Porous Media, $3^{\text {ra }}$ ed. Toronto, Canadá: University of Toronto Press, 1974 , cap. 9 , pp. 215-255.

[18] B. Ghanbarian, A. G. Hunt, R. P. Ewing, y M. Sahimi, "Tortuosity in Porous Media: A Critical Review," Soil Sci. Soc. Am. J., vol. 77, no. 5, pp. 14611477, 2013. doi: 10.2136/sssaj2012.0435

[19] E. A. Moreira, M. D. M. Innocentini, y J. R. Coury, "Permeability of ceramic foams to compressible and incompressible flow," J. Eur. Cer. Soc., vol. 24, no. 1011, pp. 3209-3218, 2004. doi: 10.1016/j.jeurceramsoc.2003.11.014

[20] M. R. Malcolm, "The Theory of Quantitative Log Interpretation", en The Fundamentals of Electric Log Interpretation, $2^{\text {da }}$ ed. New York, USA: Academic Press INC, 1957, pt. 1, pp. 1-19.

[21] F. A. Dullien, "Single-Phase Transport Phenomena in Porous Media", en Porous Media: Fluid transport and pore structure, $1^{\text {ra }}$ ed. Londres, Inglaterra: Academic Press, 1979, cap. 4, pp. 157-230.

[22] J. Cembrero, C. Ferrer, M. Pascual y M. Á. Pérez, Ciencia y tecnología de materiales - Problemas y cuestiones, $1^{\text {ra }}$ ed. Madrid, España: Pearson - Prentice Hall, 2005.

[23] D. W. Taylor, "Permeabilidad", en Principios fundamentales de mecánica de suelos, $1^{\text {ra }}$ ed. D.F, México: Continental S.A, 1961, cap. 6, pp. 119-147.

[24] B. M. Das, "Conductividad Hidráulica", en Fundamentos de ingeniería geotécnica, $4^{\text {ta }}$ ed. Santa Fe, México: Cengage Learning, 2015, cap. 6, pp. 116-141.

[25] D. Graux, "Permeabilidad y caudal", en Fundamentos de Mecánica del Suelo, proyecto de muros $y$ cimentaciones, $2^{\text {da }}$ ed. Barcelona, España: Editores Técnicos Asociados, S.A, 1975, cap. 14, pp. 355-404.

[26] G. S. Armatas, C. E. Salmas, G. P. Androutsopoulos, and P. J. Pomonis, "The effect of surface functionalization of mesoporous silicas with propylimidazol on porosity, pore connectivity and tortuosity," Stud. Surf. Sci. Catal, vol. 144, pp. 299-306, 2002. doi: 10.1016/S0167-2991(02)80148-3

[27] S. Kam et al., "Permeability to water of sintered clay ceramics", Appl. Clay Sci., vol. 46, no. 4, pp. 351357, 2009. doi: 10.1016/j.clay.2009.09.005 